\title{
Gérard Genette de Bardadrac a Postscript
}

\section{Gérard Genette from Bardadrac to Postscript}

\author{
Pedro PARdo Jiménez \\ Universidad de Cádiz \\ pedro.pardo@uca.es
}

\begin{abstract}
Known to that point for his poetics and aesthetics studies, Gérard Genette publishes in 2006 Bardadrac, a sort of informal dictionary where he picks up childhood and youth memories, anecdotes from his personal and professional life, travel experiences, reflections on contemporary society, ideas about art and literature, opinions about the most varied subjects, inventories of special linguistic usages, etc. This book begins an autobiographical series which will be completed along the following decade with four further volumes-Codicille (2009), Apostille (2012), Épilogue (2014) y Postscript (2016) - where the writer reveals his less known side in a brilliant exercise of memory, intelligence and sensitivity.
\end{abstract}

\section{Key-words}

autobiographical writing, memory, humor, aesthetics

\section{Résumé}

Connu jusque-là pour ses études de poétique et d'esthétique, Gérard Genette publie en 2006 Bardadrac, une sorte de dictionnaire informel dans lequel sont rassemblés des souvenirs d'enfance et de jeunesse, des anecdotes de la vie professionnelle et personnelle, des expériences de voyage, des réflexions sur la société contemporaine, des idées sur l'art et la littérature, des opinions sur les sujets les plus divers, des inventaires d'usages linguistiques particuliers, etc. Ce livre est le premier d'une série auto(bio) graphique qui, au cours de la décennie suivante, se complète par quatre volumes -Codicille (2009), Apostille (2012), Épilogue (2014) et Postscript (2016)-dans lesquels l'auteur nous fait connaître son côté le plus inconnu dans un brillant exercice de mémoire, d'intelligence et de sensibilité.

\section{Mots-clés}

Écriture autobiographique, souvenir, humour, esthétique. 
Moi qui disait jadis réservé, voire 'renfermé', ma vie, mes goûts, mes rêves et mes états d'âme sont devenus, plus ou moins de mon fait et sous un voile de semi-fiction, un livre ouvert que viennent à feuilleter [...] ces quelques rares lecteurs bénévoles dont l'indulgence me confond toujours (Genette, 2014: 137).

En 2006, después de una larga trayectoria en la que había transitado sucesivamente por la crítica, la poética y la estética, Gérard Genette sorprendió a sus lectores habituales con la publicación de Bardadrac, una especie de diccionario informal en el que se recogen entradas de contenido muy diverso: recuerdos de infancia y juventud, reflexiones sobre el arte y la literatura, opiniones sobre hechos actuales, inventarios de los objetos más dispares, registros de usos lingüísticos peculiares individuales y colectivos, anécdotas de la vida académica, citas humorísticas, etc. Es precisamente de su carácter heteróclito de donde deriva el extraño título del conjunto, que el autor explica en estos términos:

Par ce vocable de sa façon, Jacqueline désignait un sac aussi vaste qu'informe [...] qui contenait trop de choses pour qu'elle pût jamais y en trouver une seule. Mais la certitude trompeuse qu'elle y était la rassurait, et le mot s'appliquait par métonymie à toute espèce de désordre [...]. Il devait rester dans l'idiome de la famille, et même un peu du village (Genette, 2002: 25) ${ }^{1}$.

La gestación del libro remite originalmente a la fusión de dos proyectos independientes que Genette había ido hilando durante mucho tiempo: un repertorio satírico de expresiones e ideas convencionales extraídas mayoritariamente de los medios de comunicación y un stock de recuerdos personales que fue ganando amplitud progresivamente. El resto es un simple proceso de acumulación favorecido por el paso de los años:

Il [le livre] est, en fait, résulté d'une sédimentation, sur bien des années, de stromates de toutes sortes et de toutes provenances. Il contient par exemple un bref poème écrit vers 1947, et une chanson entendue mille fois par été dans les années cinquante, et quelques pages rescapées de la fin des années soixante-dix [...]. Une autre, qui évoque une visite à sa maison de campagne au bord de l'Adour, a paru en novembre 2002 dans le catalogue d'une exposition consacrée à Roland Barthes. Tout le reste s'est déposé sans destination ni projet pendant une vingtaine d'années, sur des feuillets plus ou moins rangés selon la nature de leur propos : biographèmes [...] authentiques ou reconstitués, observations et opinions diverses, brèves de tiroir, dérives en cascades, dérapages plus ou moins contrôlés, bestiaire fantaisiste, collection de "mots-chimères", recueil de Souvenances [...], et répertoire à la Flaubert, ou à la Proust, de cuirs, de clichés et d'idées reçues (Genette, 2009: 221).

1 Posteriormente, en Codicille, Genette se extiende sobre los posibles étimos del vocablo "Bardadrac": "Le mot [...] est une sorte d'onomatopée composite, mixte évident du vieux bric-à-brac et de l'à peine plus récent barda militaire, lui-même dérivé du mot arabe barda' $a[\ldots]$ encore en vigueur dans tout le Proche-Orient, où il désigne un bagage en fouillis" (Genette, 2009: 216-217). 
En suma, cuando decide dar el paso inaugural de la teoría a la práctica de la literatura, Genette -que tenía ya más de setenta años- lo hace acudiendo a la fórmula más inmediata que tiene a su alcance, esto es, limitándose a recuperar y en su caso a reescribir documentos otrora abandonados que constituyen fieles fragmentos de su universo personal. De ahí la frase de Jean Santeuil que se cita en el avant-propos: "Ce livre n'a jamais été fait, il a été récolté" (Genette, 2006: 7).

Sabemos sin embargo que Bardadrac no es un texto aislado. Algunos años después se publica Codicille, añadido de vocación en principio estrictamente metatextual y autocrítica que, a lo largo de su elaboración, y en virtud de las necesidades expresivas del autor, no tardó en convertirse en continuación de pleno derecho. Finalmente, y siguiendo esta misma disposición tuilée en la que cada libro se apoya en los anteriores sin por ello dejar de aportar material nuevo, lo que Genette llama la suite bardadraque vendrá a completarse sucesivamente con Apostille (2012), Épilogue (2014) y Postscript (2016), último libro publicado en vida del autor.

Si bien pueden encontrarse algunas reseñas aisladas sobre estos títulos ${ }^{2}$, la suite bardadraque no ha sido objeto por el momento de ningún estudio monográfico de conjunto, sin duda porque se trata de una obra reciente. El objetivo de las páginas que siguen es precisamente ofrecer una primera aproximación que aporte una visión global de la serie y, como tal, sirva de punto de partida para posteriores investigaciones. Abordaremos pues en primer lugar la cuestión genérica, asociada esencialmente a las peculiaridades formales del texto y a su ubicación dentro de los escritos de no ficción. En este punto nos remitiremos fundamentalmente a lo señalado por el propio autor, quien, en su calidad de poéticien, conoce y revisa exhaustivamente cada uno de estos extremos. Más tarde atenderemos a los elementos motivacionales que se encuentran en el origen de la escritura personal de Genette y, por último, a las principales redes temáticas que se despliegan a lo largo de los cinco volúmenes que componen esta suma autográfica.

Formalmente, Bardadrac se presenta como un diccionario, y en este sentido es el propio Genette quien se remite a la obra de algunos predecesores ilustres como Voltaire (Dictionnaire philosophique), Flaubert (Dictionnaire des idées reçues) o Ambroise Bierce (Dictionnaire du Diable). Sin embargo, es un hecho que Bardadrac y los textos que le siguen ${ }^{3}$ se alejan considerablemente del espíritu de un diccionario convencional, fundamentalmente porque utilizan el orden alfabético como una simple coartada:

2 Aunque están recogidas en el capítulo final de "Reseñas bibliográficas", a continuación precisamos la correspondencia exacta para cada libro: Bardadrac (Cerisuelo, 2007 y Montalbetti, 2012); Bardradrac, Codicille y Apostille (Baudry, 2013); Épilogue (Constantinescu, 2014) y Postscript (Cerisuelo, 2018).

3 Nos referimos aquí a Codicille y a Apostille. En Épilogue y Postscript, los dos últimos textos de la serie, Genette abandona la forma del diccionario por la escritura simplemente fragmentaria, separando los diferentes pasajes con el signo del asterisco. 
Bardadrac (et sa présente suite) partage donc avec les dictionnaires proprement dits un ordre alphabétique générateur de désordre thématique, qui déconstruit la réalité observée, remémorée ou fantasmée en l'indexant sur des mots-entrées [...] qui traitent cette réalité de manière à éviter toute continuité narrative ou discursive, et toute contrainte chronologique (Genette, 2009: 218) ${ }^{4}$.

Se aprecia aquí sobre todo la influencia de quien ya había esbozado un razonamiento muy similar en su Roland Barthes par Roland Barthes:

L'ordre alphabétique efface tout, refoule toute origine. Peut-être, par endroits, certains fragments ont l'air de se suivre par affinité; mais l'important, c'est que ces petits réseaux ne soient pas raccordés, c'est qu'ils ne glissent pas à un seul et grand réseau qui serait la structure du livre, son sens (Barthes, 2002: 722).

Al mismo tiempo, la serie ignora por completo la habitual coherencia entre la designación de la entrada -que el autor decide a posteriori de manera arbitraria- y la materia que en ella se trata, que a menudo resulta muy diferente. Esta inversión de la lógica entre la nominación y la definición tampoco es completamente novedosa, pues remonta igualmente a un Barthes que, en su diccionario personal, la había utilizado y explicado en estos términos: "Il ne définit pas un mot, il nomme un fragment; il fait l'inverse même d'un dictionnaire: le mot sort de l'énoncé, au lieu que l'énoncé dérive du mot" (ibid.: 720).

Por otra parte, y en razón de su carácter fragmentario y diverso, Genette sitúa la saga en la intersección de algunos géneros antiguos, en su mayoría menores y no siempre literarios -la satura latina, la fatrasie medieval ${ }^{5}$ o el essai-, y en la órbita de ciertas prácticas contemporáneas: Bardadrac es un título no sólo temático, sino también remático -esto es, que designa el género del libro-, y en este sentido la serie es homologable a otras anteriores de denominaciones similares como Variétés (Valéry), Prétextes (Gide), Approximations (Charles Du Bos), Situations (Sartre), etc.

Diccionario, miscelánea, essai... atribuciones todas válidas y sin embargo insuficientes en la medida en que no abarcan explícitamente el factor auto(bio)gráfico, que justifica el estudio de la serie en el presente volumen y que tiene una presencia más que relevante, pues Bardadrac, como los libros que le suceden:

[...] relève aussi, partiellement et dans le détail, de quelques pratiques [...] comme l'autobiographie [...], mais il s'y rattache par une relation de flirt, selon la définition

4 Ver, en el mismo sentido, Genette, 2014: 30-32.

5 Más tarde Genette insiste en que palabra satura designaba originalmente un "plat garni de toute espèce de fruits et de légumes, une sorte de macédoine; ou un ragoût, un pot-pourri ; ou une farce" (Genette, 2009: 261), reivindicando de paso el inventario completo de las metáforas literarias procedentes del lenguaje gastronómico que designan la diversidad -salmigondis, mesclun, ratatouille- hasta llegar a "zarzuela", especialmente apreciada por el autor en sus doble vertiente culinaria y musical. 
ancienne de cette pratique, qui disait en d'autres termes que l'on tourne autour de la chose sans y entrer tout à fait (Genette, 2009: 222).

También en este punto, analizado con insistencia en numerosas entradas de la serie (ver El Sérafi, 2017: 241), gusta el autor de situarse en un terreno ambiguo. Así, aunque alude desde el principio al elemento autobiográfico ${ }^{6}$, el texto renuncia por completo a una eventual veracidad absoluta, horizonte no deseado por el autor y, muy a menudo, deslegitimado como posibilidad efectiva ${ }^{7}$ :

En même temps, je vois l'idée même du roman (devenue) quasi incontournable dans la perception, dans la conduite, et surtout dans la compréhension rétrospective de chaque existence individuelle par son propre acteur-spectateur. Même Frédéric, même Félicité, pourrait dire, comme s'en prive rarement $\mathrm{M}^{\mathrm{me}}$ Michu: "Ma vie est un roman". Mais j'en trouve chez Jean-Benoît Puech une formulation plus subtile [...]: "Chacun ne connaît de sa vie que le roman qu'il s'en fait" (Genette, 2012: 275).

Nada extraño, pues la oscilación entre lo real y lo inventado es condición misma del proyecto auto(bio)gráfico, que, como afirmara Georges Gusdorf, exige no tanto la veracidad como la sinceridad:

Il [...] faut renoncer au préjugé de l'objectivité [...]. Une autobiographie ne saurait être un pur et simple procès verbal de l'existence [...] dans le cas de l'autobiographie, la vérité des faits apparaît subordonnée à la vérité de l'homme, car c'est d'abord l'homme qui est en question [...]. La signification de l'autobiographie doit donc être cherchée par-delà le vrai et le faux (Gusdorf, 1971: 232-233).

Una idea en la que coincide con su habitual antagonista Philippe Lejeune:

Une mise au point s'impose: ce qui distingue l'autobiographie du roman, ce n'est pas une impossible exactitude historique, mais seulement le projet, sincère, de ressaisir et de comprendre sa propre vie. C'est l'existence d'un tel projet qui importe, et non une sincérité à la limite impossible (Lejeune, 1971: 28).

De lo que se trata pues es de dar rienda suelta a la diversidad del yo sin reconstruirlo objetivamente, y para ello el primer paso es optar por la única modalidad autográfica que permite escapar a la cronología:

6 La cuestión de la autobiografía aparece ya, aunque de manera irónica, en “Absolu concret”, la cuarta entrada de Bardadrac -es decir al principio de la serie-, en la que podemos leer: "Mais ceci est une autre histoire, et je ne suis pas ici pour raconter ma vie. Quoique" (Genette, 2006: 11).

7 Ver por ejemplo Genette 2014: 133 y Genette, 2016: 71. No en vano es el propio Genette quien sugiere a Bernard Comment la publicación del conjunto en "Fiction \& Cie", colección de la editorial Seuil que alberga títulos tanto de ficción como de no ficción.

8 Como sehabrá notado, la idea que aquí se defiende no es más que una variante de lo que Paul Ricœur llamara la "identidad narrativa" (Ricœur, 1988). 
Mais l'écriture (peu ou prou) autographique peut adopter à l'égard du temps, je l'ai dit, trois modes stratégiques: le premier (autobiographique au sens le plus canonique) suit plus ou moins fidèlement, par récit rétrospectif et continu, l'ordre chronologique du vécu (le sous-genre des Mémoires est en principe une variante plus volontiers consacrée à la vie publique [...]); le deuxième, celui du Journal ("intime" ou non) est ou veut sembler asservi, comme son nom l'indique, au temps quotidien [...]; le troisième -l'abécédaire, bien sûr [...]- est le seul qui ne soit en rien subordonné à la temporalité d'une existence qu'il fragmente en éclats chronologiquement libres et pour ainsi dire suspendus (Genette, 2014: 32).

No en vano Genette prefiere utilizar el término "autographique" en lugar de "autobiographique": "J'esquive le bio qui m'engagerait sur le chemin de ce récit rétrospectif et continu dont la seule idée me fait mourir d'ennui" (Genette, 2012: 131) 9 .

En última instancia, estas dos últimas citas nos aportan una pista indirecta pero sustancial en la atribución del género, pues, como señala Michel Beaujour, la abdicación de la cronología es precisamente el elemento que convierte la autobiografía en autorretrato:

Celui-ci [l'autoportrait] tente de constituir sa cohérence grâce à un système de rappels, de reprises, de superpositions ou de correspondances entre des éléments homologues et substituables, de telle sorte que sa principale apparence est celle du discontinu, de la justaposition anachronique, du montage [...] La formule opératoire de l'autoportrait est donc : 'Je ne vous raconterai pas ce que $\mathrm{j}$ 'ai fait, mais je vais vous dire qui je suis' (Beaujour, 1980: 9).

La forma del diccionario parece responder por completo a esta definición. En cualquier caso, Genette es perfectamente consciente de que el proyecto que ha emprendido en sus últimos años consiste en desvelar ante sus lectores un yo si no íntimo, al menos personal, ajeno a su imagen pública y por tanto desconocido hasta ahora, un yo que es él mismo y al tiempo es otro:

Un seul (pré)nom peut désigner plusieurs personnes distinctes, ni tout à fait la même ni tout à fait une autre, et la même personne être désignée par plusieurs noms [...]. Je ne prétends échapper moi-même à ce sort ni comme "personnage" ni comme "auteur" (Genette, 2014:84-85).

Cabe preguntarse por qué en un momento dado Genette, que hasta entonces había rehuido cualquier referencia a su propia persona, decide emprender el camino de la escritura autobiográfica. La respuesta no es fácil, fundamentalmente porque el autor, que analiza

9 Dicho esto, aunque Genette renuncia categóricamente a la autobiografía convencional, no reniega en absoluto de lo autobiográfico o, si se quiere, de la autobiografía en su sentido más amplio, que extiende su jurisdicción a la suma de los documentos relativos a un autor "L'autobiographie de Victor Hugo ne se réduit pas aux recueils de notes, carnets et Choses Vues, journal de voyage sur le Rhin et autres écrits rédigés à la première personne, y compris les Contemplations et l'Art d'être grand-père. Toutes les œuvres majeures de Hugo doivent être considérées comme des 'métaphores' de Hugo” (Gusdorf, 1991: 245). En este sentido, ver Genette, 2012: 27. 
pormenorizadamente otros temas relativos a Bardadrac como el género o la componente autobiográfica, no gusta de extenderse sobre esta cuestión. Si acudimos al epitexto más o menos reciente, la única declaración que encontramos es la siguiente, extraída de una de las raras entrevistas que concedió tras la publicación de Codicille: "Tout se passe comme si à un certain moment je m'étais dit: 'Eh bien, ta subjectivité, dont tu fais grand cas, il faudrait peut-être en dire plus, il faudrait peut-être la décrire, il faudrait peut-être la mettre noir sur blanc'" (L'aire d'U, 2010), lo que no nos lleva demasiado lejos. Dentro ya de la propia serie autográfica, las referencias de Genette a los motivos que le llevan a adentrarse en la escritura personal son tan escasas como breves. Muy episódicamente alude a lo que suele denominarse la "función terapéutica" de la escritura, definiendo su actividad como una práctica egotista y “sans autre visée que d'expression de soi, au sens où 's'exprimer' est une façon de s'expulser, et donc peut-être (mais ce serait trop beau), de s'oublier" (Genette, 2014: 94) ${ }^{10}$. Más tarde insiste - con elegante aunque exagerada modestia- en que el principal y único beneficiario de su actividad es él mismo:

De ces révélations douteuses, il se fait que mes proches, déjà au clair, n'ont guère besoin, et que les autres n'en ont cure. Émises en toute inutilité publique, le plus intéressé en est certes l'émetteur lui-même, qui y trouve moyen de s' "étudier" en s'exprimant, de "faire le point" de temps à autre, comme on tapote un baromètre pour s'assurer d'une prévision souvent incertaine (ibid.: 137).

Se deriva aquí de lo gnoseológico a lo existencial, tanto más cuanto que lo que la imagen del barómetro sugiere es el deseo no ya de conocerse, sino también de reconocerse, la necesidad, más que legítima en una edad avanzada, de escribir no tanto para recuperar el tiempo perdido -aunque también- como para verificar que aún queda tiempo para escribir. En este sentido es inevitable evocar la entrada "Mémoires" de Codicille: "Parlant au cardinal de Retz alors retiré de toutes affaires, même d'Église, Turenne l'approuvait d'avoir ainsi mis 'quelque temps entre la vie et la mort', et se promettait de suivre cet exemple" (2009: 175-176).

Por último, afirma también Genette que cuando confía al papel sus opiniones, sentimientos y anécdotas lo hace "sans autre fonction que de "sauvegarde"” (ibid.: 67), frase que remite a un propósito testimonial como mínimo amortiguado, tanto más cuanto que el autor no pretende (re)componer ni un fresco social o histórico ni una galería de hombres ilustres. La única sección que se aproxima explícitamente a algo similar es la de "Souvenances", destinada a consignar recuerdos cotidianos, y que tiene pretensiones bastante más modestas:

10 Una idea que ya Amiel, autor admirado por Genette, había expresado de modo casi idéntico: "Pourquoi? Parce que analyser le sentiment, c'est le dissoudre, et surtout parce que l'exprimer, c'est à la lettre le sortir de soi (ex-primere)" (cit. por Cuasante, 2018: 32). 
Ma raison pour lui faire un sort particulier, quoique restreint, dans un contexte un peu plus largement voué, entre autres, a l'évocation de souvenirs, c'est, d'abord, que ceuxci relèvent plus souvent d'un (menu) passé historique, et qui "fait époque"; ensuite, que la formule [...] "Je me souviens", désigne moins un objet que le fait de s'en souvenir. Son propos spécifique est la mémoire individuelle de ce passé collectif (Genette, 2006: 394).

Sin duda una parte importante de la serie Bardadrac es la ocupada por los recuerdos de experiencias relativas a distintos periodos de la vida del autor. La infancia desde luego, de la que Genette -siguiendo la senda de Proust, como sabemos uno de sus mayores referentes literarios- extrae imágenes y episodios en los que la memoria de la familia se asocia con una afectividad poco nostálgica ${ }^{11}$ y casi siempre optimista. Así, por poner sólo un ejemplo, en la entrada "Radio days":

Comme tous les natifs de 1930 et environs, j'appartiens à la "génération radio" -la seule en son genre, puisque seule, de part et d'autre de l'Atlantique, à avoir grandi sous l'empire exclusif de ce media-là: avant nous, il n'existait guère, et mal; après nous vinrent les générations télévision [...]. A la radio des années trente, donc, sur petites ou grandes ondes $[\ldots]$, je me souviens entre autres de certains matches de boxe, crochet du droit, direct du gauche, uppercut, corps à corps, arête de l'arbitre, deuxième round, crochet du gauche, etc. jusqu'au jet d'éponge ou coup de gong final. Mon père alors se levait, éteignant aussitôt le poste par mesure d'économie, et commentait en connaisseur: "Beau match" (ibid.: 361).

No obstante, el discurso de la rememoración adopta a veces un tono más melancólico, sobre todo cuando alude a amistades perdidas e irrecuperables como la de Agustín Alberro : "Cette amitié naufragée fait maintenant partie d'une série de relations avortées, autant de fiascos affectifs dont je me demande toujours si je sus coupable ou victime. Les deux, sans doute, comme bien d'autres choses" (Genette, 2012: 18).

También hay una mirada distanciada, aunque menos emotiva y más crítica, a los años de universidad, en los que Genette se entregó con pasión desmedida a la militancia comunista, y que quedan particularmente bien reflejados en tres entradas de Bardadrac: "Concept", "Réfutation" y "Transition”. Nos encontramos aquí con un Genette ahora ya descreído y sin embargo jovialmente autocrítico, capaz en cualquier caso de asumir los errores pasados con resignación pero sin resentimiento ${ }^{12}$. Así recuerda por ejemplo un encuentro con Jean-François Lyotard:

Il m'écoutait avec intérêt, jusqu'au moment où [...] je lui signalai qu'avant ce saut fatal j'avais été moi-même un peu tenté par le trotskisme [...]. Pointant sur moi un œil devenu franchement sévère, il conclut à peu près: "En somme, si je comprends bien, tu

11 Recordemos que la entrada "Nostalgie" queda definida como "Regret stérile d'un passé imaginaire" (Genette, 2006: 303).

12 Sobre este aspecto, así como sobre los temas dominantes de Bardadrac, ver Cerisuelo, 2007. 
as été d'abord trotskiste, puis stalinien?". Cet ordre de succession avait apparemment quelque chose d'inconcevable et, pour le coup, tout à fait répréhensible [...]. Mais ce reproche implicite me choqua à mon tour. J'admets volontiers qu'on critique mes sottises, mais pas trop qu'on me dise dans quel ordre j'aurais dû les faire (ibid.: 436).

De modo similar, el retrato de la vida intelectual adulta queda plasmado en un corpus extenso que abarca comentarios y situaciones a menudo cómicas, acontecidas al propio autor o a ilustres colegas en un medio universitario retratado siempre con ironía. En él se incluyen, por ejemplo, la definición que abre la entrada "Colloques" -“Succession réglée de soliloques, bizarrement baptisés 'communications', en principe relatifs à un sujet commun, prétexte flasque à toutes digressions" (ibid.: 76) - o también el sabroso relato de un congreso organizado en 1968 en Zurich por Paul de Man:

De Man avait logé tout son monde dans un charmant hôtel de la vieille ville, mais faute de place il nous avait serrés, Jacques [Derrida] et moi, dans la même chambre à deux lits. Jusqu'ici, rien d'alarmant; c'est au moment de l'extinction des feux que mon cothurne d'un soir s'avisa qu'il avait oublié son pyjama -mais non, heureusement, sa machine à écrire portative. Ceci compensant cela, il me demanda si le bruit de son travail risquait de me gêner. Sur ma réponse forcément conciliante, il occupa une bonne part de sa nuit, et de la mienne $[\ldots]$ à taper $[\ldots]$ une communication dont $[\ldots]$ j'aurais pu inférer la teneur de la sonorité, acoustiquement différenciée, des touches de son clavier. Et je ne dis rien de la fine clochette de son chariot, détail d'époque qui n'évoque pas grand-chose aux générations numériques, qui ne savent pas ce qu'elles ont, musicalement parlant, perdu au change. Le lendemain matin, De Man, frappé de ma mine chiffonnée, me demanda si tout se passait bien. Croyant le mettre à l'aise, je répondis bravement : "Bah, à la guerre comme à la guerre". Ce n'était apparemment tout à fait rassurant, car il répliqua, dans un anglais plutôt pincé : "You are not very helpful!" (ibid.: 78-79).

Con todo, la serie Bardadrac no es sólo una mirada al pasado. En primer lugar, porque muchas de sus entradas remiten a hechos contemporáneos, no en vano el autor trata a algunos de sus personajes como eventuales interlocutores:

Dans Bardadrac et ses suites, certaines entrées, cryptées ou non, sont des sortes de "messages personnels" [...]. D'autres entrées, encore plus actives et, me semble-t-il, devenues un peu plus nombreuses dans Apostille, voulaient agir comme autant de "test", ou d'épreuves; de tel épisode vécu au passé ou revécu au présent, l'écriture et la publication visaient à provoquer ailleurs une réaction (Genette 2014: 65-66).

En segundo lugar, porque una parte importante de la serie no procede de la rememoración, sino de la simple expresión de ideas, sentimientos y preferencias personales de todo orden. Cuando de esto se trata, la fórmula de escritura que Genette emplea de manera más contundente es la de la lista. La práctica del inventario tiende a satisfacer en efecto la "pulsión taxinómica" - la expresión es de Montalbetti (Montalbetti, 2012)- de Genette, más que familiar para los lectores de su obra anterior y, desde luego, para él mismo, quien no duda en 
reivindicarla en forma de "libido classificandi" (Genette, 2009: 145). Así, en la serie pueden encontrarse extensos listados sobre los temas y objetos más peregrinos -falsas buenas ideas, museos dedicados a un artista único, materias textiles, etc. ${ }^{13}$-, algunos de ellos presentes ya en Bardadrac y aumentados en volúmenes posteriores de la serie, como los de las entradas "Bestiaire", "Cauchemars préférés", "Ponts" y, desde luego, "Cuirs", que da cabida a hilarantes deformaciones lingüísticas:

Involontaires ou délibérés, j'augmente ici ma collection: "Dénégation de compétence" (faux-fuyant de responsabilité), "Avoir de la fuite dans les idées" (constat de sénescence) "Apprendre ou à laisser" (sélection par l'échec), [...] "Talent d'Achille" (en principe, il s'agit de devancer la tortue) (ibid.: 84$)^{14}$.

El sentido del humor recorre igualmente dos listados satíricos más extensos, cuya presencia sistemática en los tres primeros volúmenes los convierte en secciones independientes, a su vez ordenadas alfabéticamente. Hablamos de "Médialectes", amplísimo glosario -54 páginas sólo en Bardadrac- del peculiar uso de la lengua que se hace en los medios de comunicación:
Anonymes. Parmi la foule des anonymes, on reconnaît quelques personnalités. En fait, on reconnaît aussi très bien les anonymes, à ce signe infaillible qu'on ne les reconnaît pas $[\ldots]$.
Arrêter. Interdit en forme d'injonction positive. Ne dites plus "Il ne faut pas vendre la peu de l'ours" mais "Il faut arrêter de vendre la peu de l'ours". En somme, il faut arrêter de dire "Il ne faut pas..." et commencer de dire "Il faut arrêter de..." [...].
Bien. Certain philosophe un peu (pas trop) médiatique ponctue littéralement son dis- cours d'un "bon" hautement récurrent : c'est sa virgule (Genette, 2006: 224-227).

Y de "Mots-chimères", inventario de neologismos en forma de calambur que se obtienen de la fusión de dos palabras diferentes, "hybrides verbaux dont chaque élément contribue, plus ou moins efficacement, au sens synthétique de l'ensemble" (ibid.: 288). Tras recordar que se trata de un juego verbal anteriormente practicado por grandes escritores como los Goncourt o Joyce, Genette elabora su propia cosecha, de la que extraemos sólo una muestra:

Adulescent. Post-ado.

Alambigu. Contourné jusqu'à l'équivoque.

Alibicyclette. Excuse invoquée pour une infraction commise à vélo [...].

Amnéricain. Citoyen des États-Unis dépourvu de mémoire.

Analphabétisation. Régression en cours au stade oral de la culture.

Anarchévêque. Prélat libertaire (ibid.: 289).

13 Para una "meta-lista" de las principales listas de los tres primeros volúmenes de la serie ver Baudry, 2013.

14 “J'aime bien aussi les simili-cuirs: j'entends par là des locutions, à ma connaissance parfaitement correctes, que certains croient devoir amender en y dénonçant une étymologie populaire. Ainsi, 'cor anglais' serait un cuir pour cor anglé" (Genette, 2006: 111). 
En los dos últimos volúmenes de la serie, sin embargo, la sustitución del orden alfabético por la simple escritura fragmentaria permite al autor avanzar libremente por asociaciones sucesivas y, con ello, abandonarse a reflexiones más prolongadas, cosa que sucede, por ejemplo, en las más de treinta páginas de Épilogue dedicadas al tiempo y a la memoria (ver Genette, 2014: 32-65) o en Postscript, donde encontramos un análisis teórico de las categorías de género y estilo que atraviesa la literatura, la música y la pintura (ver Genette, 2016: 146-168).

$\mathrm{Y}$ es precisamente el arte, en todas sus formas y modalidades, el tema que más prolijamente trata Genette a lo largo de la serie, tanto para defender sus ideas estéticas como para exponer sus gustos personales. A veces lo hace a partir de entradas dedicadas a autores concretos como "Wright" (Bardadrac), "Gehry" (Apostille) o "Bach" (Codicille), pero también abordando géneros o subgéneros específicos, como en "Concerto" (Apostille) en el ámbito de la música clásica o "Duo" (Apostille) en el del jazz. En ocasiones lo que se nos ofrece es un análisis comparativo, que puede tomar como objeto asuntos tan diversos como las variantes del clasicismo en las artes -"Classique" (Codicille)- o las diferentes representaciones del tema de Ariane en un poema de Catulo, un cuadro de Le Nain y la ópera de Strauss -“Ariane" (Apostille). Muy a menudo, el autor parte de un elemento o hecho puntual para posteriormente ir abriendo el foco hasta cuestiones generales, tal como sucede en "Goyas" (Codicille), entrada en la que una breve cita ajena da lugar a un discurso sobre la naturaleza de la apreciación del objeto artístico ${ }^{15}$.

Nos detendremos un momento en esta última entrada, que ilustra a la perfección el modo en que Genette reivindica una concepción abierta del fenómeno estético:

Je tiens toujours que les "valeurs" en général sont essentiellement subjectives; [...] que les valeurs éthiques sont régulées par des obligations transcendantes argumentables; [...] mais que les valeurs esthétiques, échappant à ces normes d'obligation, sont comme telles totalement autonomes et que la prétention à l'universalité du jugement de goût n'est que l'effet d'une illusion [...]. D'exprimer de temps en temps cette conviction obstinée m'a parfois fait considérer comme un relativiste absolu pur qui "tout se vaut" (Genette, 2009: 125).

Que el juicio sea libre y subjetivo no significa que la apreciación del arte escape al análisis y a la argumentación, fundamentalmente porque se basa en una objetivación posterior susceptible de ser aceptada colectivamente:

\footnotetext{
En matière d'œuvres, c'est-à-dire de productions humaines, un jugement peut être plus "éclairé" (mieux informé) qu'un autre, par exemple, sur des données historiques, génétiques ou génériques, mais il ne s'agit plus alors d'une appréciation purement esthétique, mais artistique, c'est-à-dire appliquée à un champ défini par les paramètres (historiques, génériques...) propres à telle ou telle forme d'art (ibid.: 126).
}

15 Ver también las entradas citadas por Cerisuelo, 2007: 768. 
Dentro ya del universo de la escritura, esta concepción abierta de lo artístico queda resumida en algunas ideas que Genette ya había plasmado en Fiction et diction y en las que ahora insiste sin descanso, probablemente porque constata que no han encontrado el eco esperado: para definir un obra como "literaria" podemos servirnos del criterio -aristotélicode la ficción, pero también del criterio de la dicción, que permite enjuiciar la literariedad condicional de un texto a partir de la calidad de la escritura y, con ello, incluir dentro de la esfera de lo literario los géneros de no ficción (ver Genette, 1991). En este sentido, son muy numerosos los pasajes en los que Genette lamenta la reducción de la Literatura al campo exclusivo de la novela:

En tout cas, la vulgate du "tout roman" qui triomphe aujourd'hui -par réduction implicite de la littérature au mode de la fiction, puis de celui-ci au genre du roman promu, comme on l'a dit, "synecdoque de la littérature tout entière"- m'apparaît souvent comme une maladie sénile de la culture occidentale, ou peut-être, "mondialisation" aidant, de la sous-culture universelle (Genette, 2009: 249) ${ }^{16}$.

Y, con ella, la exclusion de los géneros no narrativos -“Au temps pour Montaigne, pour Pascal, pour La Bruyère, pour Saint-Simon et pour quelques autres, dont, au passage, tous les poètes, et les dramaturges: adieu Racine, adieu Shakespeare" (Genette, 2006: 352) y de los géneros de no ficción, tanto más dolorosa cuanto que son estos géneros los preferidos del autor:

Le fait est que je donnerais volontiers non seulement, comme tout un chacun, la Nouvelle Héloïse pour une (certaine) page des Confessions, et bien entendu Les Natchez, Atala et René pour à peu près n'importe quelle autre des Mémoires d'outre-tombe ou de la Vie de Rancé, mais aussi, de façon peut-être un peu plus déviante, Les Misérables pour Choses vues, Madame Bovary pour quelques chapitres de Par les champs et par les grèves, et peut-être même Le Rouge et le Noir pour Rome, Naples et Florence ou les Mémoires d'un touriste (Genette, 2009: 251).

Por esta misma razón Genette recusa insistentemente la distinción, otrora propuesta por Barthes en sus Essais critiques, entre quienes escriben de manera instrumental, guiados por el deseo de comunicar una idea -écrivants-y quienes lo hacen de manera intransitiva, es decir, por el puro placer de trabajar (con) el lenguaje -écrivains. Si en Bardadrac la califica simplemente de "bien fragile" (Genette, 2006: 352), y en otros lugares de la serie se limita a expresar su desacuerdo con ella ${ }^{17}$, en Épilogue la presenta como una “distinction aujourd'hui

16 Ver también, por ejemplo, Genette, 2009: 216 y Genette, 2012: 275.

17 Ver por ejemplo Genette, 2009: 35 o Genette, 2012: 61. En realidad, el rechazo de Genette es más amplio: "De fait, non seulement je récuse la distinction ('aujourd'hui classique', comme on dit), entre écrivains et écrivants, mais je refuse, en elle-même, pour elle-même et sans raison articulable, la notion, selon moi toujours un peu vulgaire, ou scolaire, d'écrivain" (ibid.). 
rebattue" (Genette, 2014: 156), esto es, como un concepto considerado caduco definitiva y colectivamente ${ }^{18}$

En suma, la estimación del valor literario de una obra sólo puede ejecutarse apropiadamente desde un relativismo crítico que exige desacralizar la noción de "Literatura" $-\mathrm{y}$, de paso, la de "Escritor"- y, desde luego, tener en consideración el papel decisivo de la lectura, hecho que Genette ilustra una vez más apelando al Pierre Ménard de Borges:

A chaque lecture, chaque livre est mentalement réécrit par son lecteur comme Ménard réécrivit le Quichotte. Ainsi l'infatigable fable borgésienne est peut-être moins une parabole sophistiquée de la production littéraire qu'une description fidèle, somme toute évidente et peut-être banale, de l'acte de lire [...]. Et c'est peut-être ici le lieu de rappeler le célèbre mot de Pascal: “Ce n'est pas dans Montaigne, mais dans moi, que je trouve tout ce que je vois" (Genette, 2016: 43) ${ }^{19}$.

Archivo de la memoria y de la sensibilidad del Genette más personal, la serie Bardadrac constituye un recorrido emocional e intelectual que extrae su riqueza de la combinación de dos elementos sustanciales, a saber la asimilación inteligente del discurso de los otros, que el autor siempre toma como punto de partida de sus propias elucubraciones, y el sentido del humor siempre amable de quien escribe ya a cierta distancia de lo vivido y ve las cosas en su justa dimensión: "Me manquent effectivement, et, je le crains, définitivement, le sens du sacré, celui du sublime, et peut-être même celui du sérieux. Cette entrée devrait donc faire exception: mettons que je n'ai rien dit" (Genette, 2006: 197).

\section{Referencias bibliográficas}

BAUdrY, Marie. 2013. "Le Désordre du discours" in Acta fabula, vol. 14, n 4 [consultado el 26-11-2018] <http://www.fabula.org/acta/document7870.php>.

Barthes, Roland. 2002. Euvres complètes, IV. Paris, Seuil.

Beaujour, Michel. 1980. Miroirs d'encre. Rhétorique de l'autoportrait. Paris, Seuil.

Cerisuelo, Marc. 2007. “La zarzuela d'un amateur” in Critique, nº 725, 757-769.

Cerisuelo, Marc. 2018. "Derniers richochets” in Critique, no 858, 884-888.

Cuasante Fernández, Elena. 2018. "Las escrituras del yo y sus variantes funcionales" in Revista de Filología, no 37, 25-39.

EL SÉRAFI, Inès. 2017. 'D'un Moi l'autre. Les autoportraits de Roland Barthes et de Gérard Genette ou l'art de flirter avec le biographique” in Poétique, n 182, 237-258.

18 Sobre esta distinción, así como sobre su significado en la relación entre Genette y Barthes, ver Schuerewegen, 2012 .

19 Como bien recuerda Montalbetti (Montalbetti, 1998: 105-112), Genette se sirve de la fábula de Pierre Ménard para ilustrar el proceso de la lectura en varias de sus obras, entre ellas Figures (1966) y Palimpsestes (1982). 
Anales de Filología Francesa, n. ${ }^{\circ}$ 27, 2019

Gérard GenetTe de BARDADRAC A POSTSCRIPT

Genette, Gérard. 1991. Fiction et diction. Paris, Seuil.

Genette, Gérard. 2006. Bardadrac. Paris, Seuil.

Genette, Gérard. 2009. Codicille. Paris, Seuil.

Genette, Gérard. 2012. Apostille. Paris, Seuil.

Genette, Gérard. 2014. Épilogue. Paris, Seuil.

Genette, Gérard. 2016. Postscript. Paris, Seuil.

Gusdorf, Georges. 1971. "Conditions et limites de l'autobiographie” in LeJeune, Philippe. L'Autobiographie en France. Paris, Armand Colin, 217-236.

Gusdorf, Georges. 1991. Lignes de vie, 1. Les Écritures du moi. Paris, Éditions Odile Jacob.

L'aire d'U. Le webmedia de l'Université Rennes 2. 2010. "De Figures à Codicille, un entretien avec Gérard Genette". France: Université de Rennes 2 [consultado el 29-11-2018] $<$ https://www.lairedu.fr/media/video/entretien/de-figures-a-codicille-un-entretien-avec-gerard-genette/>.

Lejeune, Philippe. 1971. L'Autobiographie en France. Paris, Armand Colin.

Montalbetti, Christine. 1998. Gérard Genette. Une poétique ouverte. Paris, Bertrand-Lacoste.

Montalbetti, Christine. 2012. "Voyage en Genettie: Bardadrac, ou la taxinomie au cœur du désordre" in Acta fabula, n 9 [consultado el 26-11-2018] <http://www.fabula.org/acta/ document1428.php>.

Constantinescu, Muguraș. 2014. "Pour ne pas en finir avec Genette" in Acta fabula, vol. $15, \mathrm{n}^{\circ} 6$ [consultado el 10-12-2018] <https://www.fabula.org/revue/document8783.php>.

Ricceur, Paul. 1988. "L'identité narrative” in Esprit, n 140-141, 295-304.

Schuerewegen, Franc. 2012. “Genette devient écrivain”, Fabula-LhT, no 10 [consultado el 20-12-2018] <http://www.fabula.org/lht/10/schuerewegen.html>. 